\title{
An evaluation into the use of Procalcitonin levels as a biomarker of bacterial sepsis to aid the management of Intrapartum Pyrexia and Chorioamnionitis: a prospective observational cohort study
}

\author{
Sarah Walker ${ }^{1}$, Irasha Harding ${ }^{2}$, Kamran Soomro ${ }^{3}$, Andrew Bamber ${ }^{4}$, Sophie Carrick ${ }^{1}$, \\ Abdul Waheed ${ }^{1}$, and Rachel Liebling ${ }^{1}$ \\ ${ }^{1}$ University Hospitals Bristol and Weston NHS Foundation Trust \\ ${ }^{2}$ UK Health Security Agency South of England \\ ${ }^{3}$ University of the West of England \\ ${ }^{4}$ North Bristol NHS Trust
}

January 18, 2022

\begin{abstract}
Objective: Procalcitonin (PCT) is an established biomarker for bacterial sepsis in the non-pregnant population. We aimed to evaluate PCT levels in women and their neonates managed with suspected intrapartum bacterial sepsis, to determine if PCT could be utilised to optimise diagnosis and management. Design: Prospective observational cohort study. Setting: University Hospitals of Bristol and Weston NHS Foundation trust. Population: 117 women and their neonates managed for suspected intrapartum sepsis from June-October 2020. Methods: PCT levels were measured in addition to routine biomarkers white cell count and c-reactive protein, in women and their neonates during initial septic screen and follow up blood samples. Placentas underwent detailed histopathology. Main Outcome Measures: Maternal and neonatal parameters were used to categorise cases into: 'high-suspicion bacterial sepsis (BS),' 'equivocal BS' and 'low-suspicion BS.' Kruskal-Wallis test was performed comparing categories with biomarker values and placental histology scores. Results: PCT was raised in 6 women in the initial septic screen sample, compared to 100 with a raised CRP. There was a significant difference in maternal postnatal PCT results between 'highsuspicion BS' and 'low-suspicion BS' categories. $71.2 \%$ of placentas showed varying degrees of chorioamnionitis. Conclusions: In our cohort of women, $94.6 \%$ had normal PCT levels whilst in labour at the time of the septic screen, consistent with the low number of confirmed bacteraemia. This provides a basis that PCT may complement clinical judgement and interpretation of already utilised prognostic and diagnostic tests, in order to improve patient care in the management of intrapartum sepsis.
\end{abstract}

An evaluation into the use of Procalcitonin levels as a biomarker of bacterial sepsis to aid the management of Intrapartum Pyrexia and Chorioamnionitis: a prospective observational cohort study

S Walker, ${ }^{1}$ I Harding, ${ }^{2}$ K Soomro, ${ }^{3}$ AR Bamber ${ }^{4}$ S Carrick, ${ }^{1}$ AH Waheed, ${ }^{1}$ RE Liebling ${ }^{1}$

${ }^{1}$ Department of Obstetrics and Gynaecology, University Hospitals Bristol and Weston (UHBW) NHS Foundation Trust, Bristol, UK, BS1 3NU

${ }^{2}$ UK Health Security Agency, 2 Rivergate, Bristol, BS1 6EH

${ }^{3}$ Department of Computer Science Research Centre, University of the West of England, Bristol, UK, BS16 $1 \mathrm{QY}$

${ }^{4}$ Department of Cellular Pathology, North Bristol NHS Trust, Bristol, UK, BS10 5NB 
Corresponding Author: Dr S Walker, 10 Downend Park, Bristol, BS7 9PU,

Email: s.walker31@nhs.net. Mobile: 07756834376

Running Title: (50 characters)

PCT in Intrapartum Pyrexia \& suspected Chorioamnionitis

\section{Abstract}

\section{Objective:}

Procalcitonin $(\mathrm{PCT})$ is an established biomarker for bacterial sepsis in the non-pregnant population. We aimed to evaluate PCT levels in women and their neonates managed with suspected intrapartum bacterial sepsis, to determine if PCT could be utilised to optimise diagnosis and management.

Design: Prospective observational cohort study.

Setting: University Hospitals of Bristol and Weston NHS Foundation trust.

Population: 117 women and their neonates managed for suspected intrapartum sepsis from June-October 2020 .

Methods: PCT levels were measured in addition to routine biomarkers white cell count and c-reactive protein, in women and their neonates during initial septic screen and follow up blood samples. Placentas underwent detailed histopathology.

Main Outcome Measures: Maternal and neonatal parameters were used to categorise cases into: 'highsuspicion bacterial sepsis (BS),' 'equivocal BS' and 'low-suspicion BS.' Kruskal-Wallis test was performed comparing categories with biomarker values and placental histology scores.

Results: PCT was raised in 6 women in the initial septic screen sample, compared to 100 with a raised CRP. There was a significant difference in maternal postnatal PCT results between 'high-suspicion BS' and 'low-suspicion BS' categories. $71.2 \%$ of placentas showed varying degrees of chorioamnionitis.

Conclusions: In our cohort of women, $94.6 \%$ had normal PCT levels whilst in labour at the time of the septic screen, consistent with the low number of confirmed bacteraemia. This provides a basis that PCT may complement clinical judgement and interpretation of already utilised prognostic and diagnostic tests, in order to improve patient care in the management of intrapartum sepsis.

Keywords: Intrapartum sepsis, procalcitonin, sepsis biomarkers.

\section{Introduction}

Sepsis biomarkers have been the subject of numerous research studies; aiming to identify patients with bacterial sepsis, differentiate sepsis from other non-infectious inflammatory pathologies, predict clinical severity and guide antibiotic stewardship. The biomarkers predominantly used in the pregnant population include white-cell-count (WCC), C-reactive-protein (CRP) and lactate; all known to be nonspecific for inflammation versus infection. ${ }^{1}$

Procalcitonin (PCT) is an established marker for sepsis in the non-pregnant population, with better diagnostic and prognostic value for bacterial infections. ${ }^{2,3} \mathrm{PCT}$, a precursor of calcitonin produced by $\mathrm{C}$ cells of the thyroid gland, is involved in maintaining calcium levels in the blood and released into the circulation in response to endotoxins and pro-inflammatory stimuli, specifically those originating from bacteria. Levels are usually low in healthy people, people with viral infections, chronic inflammatory disorders or autoimmune processes. PCT increases significantly within the first hours in severe bacterial infections (latent period 2-4 hours) and has a short half-life, helping guide and monitor response to antibiotic therapy. ${ }^{3,4} \mathrm{PCT}$ use in diagnosing pregnancy-associated sepsis is relatively unexplored and reference values for PCT in pregnancy have not been established. ${ }^{5,6}$ 
Chorioamnionitis is reported to complicate $1-4 \%$ of births worldwide and is associated with significant maternal and perinatal adverse outcomes. ${ }^{7,8}$ Consequently, a low threshold to commence empirical broadspectrum antibiotics in women developing intrapartum pyrexia is advised. ${ }^{1,9,10}$ The reported incidence of intrapartum pyrexia is high, ranging from $3-7 \% .{ }^{11,12}$ The risk of neonatal sepsis in new-borns delivered from mothers with intrapartum pyrexia is low at $0.24 \%$ (rate $<1$ in 400$).{ }^{11}$

Diagnosing chorioamnionitis and managing intrapartum sepsis presents unique diagnostic difficulties and is an ongoing clinical challenge. While intrapartum pyrexia may be an indicator of chorioamnionitis, most cases are secondary to non-infectious factors including: epidural analgesia; prolonged labour; increased ambient temperature; use of prostaglandins and activation of the pro-inflammatory cascade during parturition. ${ }^{1,12}$ Furthermore, pregnancy causes physiological adaptations which mimic those of early infection, including increased heart rate and reduced blood pressure. These challenges lead to over-diagnosis and treatment of sepsis in labour. ${ }^{1}$

There is growing evidence suggesting exposure to intrapartum antibiotics is associated with alterations in infant intestinal microbiome, impacting on early immune development and increasing the risk of immunemediated diseases such as asthma, allergy and atopy. ${ }^{13,14}$

The objective of this study was to evaluate PCT levels in women and their neonates managed for suspected intrapartum sepsis, to determine if PCT could be utilised to optimise the diagnosis and management of bacterial sepsis.

\section{Method}

A prospective observational cohort study was carried out at St Michael's Hospital, University Hospitals of Bristol and Weston (UHBW) NHS Foundation trust, averaging 5000 deliveries a year. The hospital research ethics committee approved the study protocol and funding was provided by the department.

Over a 4-month period (June-October 2020) all women who developed intrapartum pyrexia and were commenced on the sepsis pathway, according to local and national guidance, ${ }^{9,10}$ were included in the study. These women routinely have samples taken for blood cultures, full blood count, urea and electrolytes, liver function, lactate and CRP. PCT levels were added to this initial sample (denoted by the term 'Septic Screen (SS)' bloods) alongside subsequent CRP samples ('Postnatal (PN)' bloods) to avoid additional sampling. The PCT results were concealed from clinicians to avoid influencing management.

At UHBW all neonates born to women treated for intrapartum pyrexia routinely have blood collected for blood cultures, full blood count and CRP, and are commenced on intravenous antibiotics for at least 36 hours. These are stopped according to repeat inflammatory marker results and clinical appearance. For this study, initial samples were also tested for PCT levels ('Time 0' bloods) alongside subsequent CRP samples ('Day 1' bloods). The PCT results were concealed from clinicians.

The PCT was measured with the Elecsys BRAHMS PCT assay, with a value $<0.25 \mathrm{ng} / \mathrm{ml}$ considered normal for non-intensive care unit (ICU) patients and a cut off value of $<0.5 \mathrm{ng} / \mathrm{ml}$ considered normal for ICU patients. ${ }^{15,16}$

Women treated for intrapartum pyrexia routinely have a urine sample, low vaginal swab (LVS) and throat swab sent for microbiology and all women in this cohort were tested for COVID-19. The placentas were sent for histopathological assessment and examined by a consultant perinatal pathologist, who scored any inflammation present using published criteria. ${ }^{17}$ The staging and grading of chorioamnionitis was scored using the following criteria; umbilical cord inflammation stage (maximum 3), umbilical cord inflammation grade (maximum 2), membranes inflammation stage (maximum 3), membranes inflammation grade (maximum 2), chorionic vasculitis stage (maximum 3), chorionic vasculitis grade (maximum 3), chorionic plate inflammation stage (maximum 3), chorionic plate inflammation grade (maximum 3), villitis (maximum 1 ) and intervillositis score (maximum 1 ); giving a maximum possible score of $24 .{ }^{17,18}$

Maternal antepartum and intrapartum records were reviewed for vital signs on admission, uterine tenderness, 
foul-smelling amniotic fluid, urine output and maximum maternal temperature prior to delivery. Additional variables were recorded such as duration of rupture of membranes, use of fetal blood sampling (FBS), use of epidural, induction of labour, route and location of delivery, Group B Haemolytic streptococcus (GBS) status and postpartum maternal length of hospital stay. Clinical markers of neonatal infection were identified by reviewing neonatal records. Specific neonatal markers included, fetal tachycardia, temperature, respiratory distress and poor feeding. Additional neonatal variables reviewed were birth weight, gender, neonatal course of antibiotics and length of stay. All information was recorded and stored on a dedicated database.

Maternal cases were divided into three categories of 'high-suspicion bacterial sepsis (BS)', 'equivocal BS' and 'low-suspicion BS,' using a composite of clinical features, sepsis in obstetrics score ${ }^{19}$ and investigation results. The neonates were also divided into three categories of 'high-suspicion BS,' 'equivocal BS' and 'low-suspicion BS,' using a composite of clinical features, neonatal early-onset sepsis calculator ${ }^{20,21}$ and investigation results. To provide triangulation the categorisation was carried out by three clinicians blinded to each other and PCT results. The histopathology of the placenta was analysed separately with the pathologist blinded to the clinical findings.

Correlations were performed between each of the biomarker values and placental score from initial and follow up samples $(0=$ no correlation, $1=$ complete correlation). The Kruskal-Wallis test was performed to correlate each clinical category with the individual biomarker values and placental histology scores. A p-value $<0.05$ was considered significant.

\section{Results}

A total of 117 women along with their neonates were included in the study ( $7 \%$ of our delivered population in the study period). A previous audit performed in the department demonstrated the rate of intrapartum pyrexia at $13.5 \%$ and we therefore estimate that we included about $53 \%$ of the cases. Exclusion criteria included incomplete data, gestation $<37$ weeks, women with immunocompromising conditions (e.g., chronic steroid use during pregnancy, transplant recipients), and known chronic infection (i.e., hepatitis B, hepatitis C).

Blood cultures were performed in $115(98.3 \%)$ women and all were taken prior to the commencement of antibiotics (see table 2). Two were positive with clinically significant organisms (1.7\%). One blood culture grew Proteus mirabilis which correlated to a raised postnatal maternal PCT of 2.6, but normal PCT in the neonate. The other blood culture grew GBS (as did microbiology for the low vaginal swab and placental swab), which correlated to a raised PCT of 3.6 in the neonate. In this case maternal PCT samples were not taken.

Urine cultures were performed in 107 women (91.5\%) and two were culture positive with Escherichia coli . LVS cultures were performed in 100 women (85.5\%) and 15 grew GBS. Of those 15, one was detected antenatally and received intrapartum prophylactic benzylpenicillin. Two had previous negative GBS swabs during their antenatal care. Throat swab cultures were performed in 86 women $(73.5 \%)$ and two were positive (Group A Streptococcus and Group G beta haemolytic Streptococcus ). Placental swab cultures were performed in 21 women (17.9\%) and two grew Escherichia coli and four grew GBS (two correlated to GBS on LVS, and one had GBS on blood culture). Covid-19 PCR swabs were performed in 107 women (91.5\%) and two were positive.

A raised PCT of [?]0.25ug/L in 'Septic Screen (SS)' blood samples was found in 6 women (5.4\%), compared to a raised CRP[?]10mg/L in 100 women $(86 \%)$ and a raised $\mathrm{WCC}>11 \times 10^{9} / \mathrm{L}$ in 112 women $(96 \%)$. A raised $\mathrm{PCT}$ in 'Postnatal (PN)' blood samples was found in 39 women $(41.5 \%$, range $0.3-38.5 \mathrm{ug} / \mathrm{L})$, compared to a raised CRP [?]10mg/L in all of the women $(100 \%$, range $13-304 \mathrm{mg} / \mathrm{L})$.

A raised PCT of [?]0.25ug/L was found in 47 neonates (55.3\%, range 0.3-4.6) at the 'Time 0' blood samples and in 95 neonates $(98.9 \%$, range $0.3-58.5 \mathrm{ug} / \mathrm{L})$ 'Day 1' blood samples. Whereas, a raised CRP of [?]10mg/L was found in 7 neonates $(5.98 \%$, range $10-58 \mathrm{mg} / \mathrm{L})$ at the 'Time 0 ' blood samples and in 41 neonates $(35.04 \%$, range $10-88 \mathrm{mg} / \mathrm{L}$ ) 'Day 1' blood samples (see Tab. 2). 


\section{Statistics}

Correlations were performed to compare values of maternal and neonatal biomarkers from initial and follow up blood samples. Where the CRP was raised for the mother and neonate on initial sampling it was likely to be raised on the second sample. There was also a positive correlation between both 'time 0' and 'day 1' PCTs with the CRP results for the neonate, +0.509 and +0.409 respectively. There was a positive correlation between maternal 'SS' PCT and neonatal 'time 0' PCT $(+0.305)$ and neonatal 'day 1' PCT $(+0.404)$. This was a higher correlation compared to maternal 'SS' CRP and neonatal 'time 0' CRP $(+0.098)$ or neonatal 'day 1' CRP (+0.141).

Table 1 shows the number of maternal and neonatal participants that were divided into the 3 determined categories. The spread of biomarker results for the maternal 'SS' and 'PN' bloods according to the three categories are illustrated in figure 1.

The mean PCT results for the 3 categories for the maternal 'PN' blood tests were $0.15 \mathrm{ug} / \mathrm{L}$ for 'low-suspicion BS,' 0.45ug/L for 'equivocal BS' and 7.26ug/L for 'high-suspicion BS.' The mean PCT results for the neonatal 'time 0' blood tests were $0.25 \mathrm{ug} / \mathrm{L}$ for 'low-suspicion BS,' $0.53 \mathrm{ug} / \mathrm{L}$ for 'equivocal BS' and $0.98 \mathrm{ug} / \mathrm{L}$ for 'highsuspicion BS.' The mean PCT results for the neonatal 'day 1' blood tests were 1.03ug/L for 'low-suspicion BS,' $5.05 \mathrm{ug} / \mathrm{L}$ for 'equivocal BS' and 8.48ug/L for 'high-suspicion BS.' When performing the Kruskal-Wallis Test between the categories there was no significant difference in maternal 'SS' PCT results between the 3 categories which would be expected as the 'SS' PCTs were only raised in 6 women (PCT[?]0.25 ug/L). However, for the 'PN' PCT results there was a significant difference between the 'high-suspicion BS' and 'low-suspicion BS' categories ( $\mathrm{p}=0.004)$. For neonates, there was a significant difference between the 'highsuspicion BS' and 'low-suspicion BS' categories for both the 'time 0' $(\mathrm{p}=0.0349)$ and the 'day 1' PCT results $(\mathrm{p}=0.008)$.

There were no significant differences between the CRP and WCC results in the 3 categories for maternal results and no significant difference between the WCC results in the 3 categories for neonatal results. However, there was a significant difference in CRP results between 'high-suspicion BS' and 'equivocal BS' for the neonatal 'time 0' $(\mathrm{p}=0.022)$ and the 'day 1' $(\mathrm{p}=0.0002)$ CRP results.

A total of 80 women $(68.3 \%)$ had completed placental histopathology and they were scored out of a total of 24 for their grading and staging of chorioamnionitis. A high proportion, 57 out of $80(71.2 \%)$, showed varying degrees of chorioamnionitis (range 5 to 18, mean score 10.6). Figure 2 shows the placental histopathology scores for the 3 maternal categories. There was a significant difference between the maternal categories of 'high-suspicion BS' to both 'equivocal BS' $(\mathrm{p}=0.02)$ and 'low-suspicion BS' $(\mathrm{p}=0.00018)$. There was a weak correlation between maternal 'PN' PCT and placental score $(+0.17)$ and neonatal 'day 1' PCT and placental score $(+0.234)$.

\section{Discussion}

\section{Main findings}

We sought to analyse PCT levels in a cohort of women who presented with possible intrapartum sepsis. We hypothesised that a small number would have a proven bacteraemia but that a proportion would display features highly suggestive of bacterial sepsis. We set out to determine if there was a relationship between PCT and bacterial sepsis. The results show a significant difference in PCT results between the 'low-suspicion BS' and 'high-suspicion BS' categories for maternal and neonatal follow up PCT results. In addition, there appears to be a linear correlation in maternal and neonatal PCT levels with maternal and neonatal clinical condition (see Fig.1). This suggests PCT could be of diagnostic and prognostic value for bacterial infections in the management of intrapartum pyrexia.

\section{Strengths and limitations}

To our knowledge this is the first time PCT has been analysed alongside the routine septic screen investigations in both maternal and neonatal patients, thus reflecting a 'real world' clinical context. We collected a 
detailed dataset for each patient, ensuring accurate interrogation of each clinical episode.

We did not anticipate such a low number of raised PCT in this cohort of women, necessitating a larger cohort to adequately power a study investigating clinical outcome with PCT levels. However, this finding is important, demonstrating that PCT does not appear to be altered by the physiology of labour, unlike other inflammatory markers in clinical use.

We chose to categorise our cases into 'low-suspicion BS,' 'equivocal BS' and 'high-suspicion BS.' This approach has been adopted in other studies. ${ }^{22}$ We were careful to include validated methods of categorisation such as 'Sepsis in Obstetrics Score' and the 'Kaiser' scoring for neonates. ${ }^{19,21}$ We also acknowledge that the routine investigation results formed part of this categorisation and so any effect may have been artificially strengthened. However, as in the case of CRP, this would have led to an enhanced correlation, where high CRP was used to denote 'high-suspicion BS,' one would anticipate a higher correlation coefficient for CRP.

\section{Interpretation}

Clinical utilisation of host-response, blood infection biomarker PCT has gained attention and has already been approved for guidance of antimicrobial therapies and antimicrobial stewardship in patients with respiratory infection and sepsis. ${ }^{23,24}$ More recently, it has been shown as an indicator of superimposed bacterial infection in coronavirus disease 2019 (COVID-19) and guided reduced antibiotic use in COVID-19. ${ }^{25,26}$ Clinical application in maternity and neonates is relatively unexplored and remains unvalidated.

All women had a SS PCT $<0.25 \mathrm{ug} / \mathrm{L}$ except 6 women (5.4\%). This supports other studies which show that $\mathrm{PCT}$ as a biomarker is not influenced by pregnancy or labour compared to the normal adult population. ${ }^{22,27,28}$ Our results show that CRP and WCC were raised in a significant proportion of women in labour, in stark contrast to the small number with a raised PCT (86\%, 96\% and 5\% respectively). Although our numbers are small, this study suggests that PCT may identify bacterial sepsis in the obstetric population with greater specificity. PCT could also be utilised as an antibiotic stewardship tool, aiding cessation of antibiotics in women with a low likelihood of infection and two negative serial PCTs.

In this study the neonates had a raised PCT of [?]0.25ug/L in $55.3 \%$ at the 'time 0 ' and in $98.9 \%$ at the 'day 1 ' blood samples. Whereas, a raised CRP of [?]10mg/L was found in $5.98 \%$ at the 'time 0 ' and in $35.04 \%$ at the 'day 1' blood samples. This study supports recent evidence suggesting a physiological rise in PCT in a well neonate. ${ }^{29}$ We demonstrated a significant increase in the neonatal 'day 1' PCT compared to their 'time 0' PCT which would not support the theory of placental transfer of maternal PCT.

Blood cultures are used to diagnose bacteraemia and identify pathogens, to provide information about type of microorganism and antimicrobial susceptibility. In this study, only two (1.7\%) blood cultures were positive with clinically significant organisms. Furthermore, when looking at the total number of blood cultures received from our obstetric population in 2020 , out of a total of 723 , only $13(1.8 \%)$ were positive. This supports the theories of low yield or low levels of bacteraemia in this population. ${ }^{1}$

Other studies have sought to address this issue with $<10 \%$ of cultures showing growth of bacteria. ${ }^{30}$ Moreover, contamination limits their specificity, as was the case for one woman in this study. ${ }^{31}$ A study by Laukemann et $\mathrm{al}^{32}$ analysed 1083 patients with suspected infection who had blood culture sampling on admission to the emergency department. Only $9.6 \%$ had positive blood cultures and PCT proved to be the most reliable predictor of blood culture positivity, being 8.5 -fold higher in positive blood cultures. The study suggested a cut-off for their PCT assay level of $<0.1 \mathrm{Ug} / \mathrm{L}$ to identify patients with a low risk for bacteraemia, avoiding unnecessary blood culture sampling could be avoided in these patients.

In our study, detailed placental histopathology found the majority $(71.2 \%)$ of placentas with varying degrees of inflammation. Placental histopathology has long been the 'gold-standard' for diagnosis of chorioamnionitis. Evidence is emerging which challenges the correlation between clinical and histological chorioamnionitis. ${ }^{33,34,35}$ A study by Smulian et al, ${ }^{34}$ looked at clinical chorioamnionitis and histologic placental inflammation in 139 pregnancies. They concluded that clinical chorioamnionitis and possible neonatal 
infection were not supported by histologic evidence of infection in $38.1 \%$ and $26.8 \%$ of cases respectively, suggesting other noninflammatory causes of signs and symptoms. Another study by Roberts et al, ${ }^{36}$ concluded $96 \%$ of histological chorioamnionitis cases occurred without infection, suggesting there may be alternative causes amongst low-risk women at term.

The presence of inflammation in a substantial proportion of the 'low-suspicion BS' group in this study could be supportive of these studies assertion that not all placental inflammation is the result of infection. An alternative, and perhaps more likely, explanation of the findings is that while inflammation in the placenta is caused by local infection, this may be limited to the placenta, cord and membranes, and does not always lead to significant infection in the mother or baby. Where inflammation in both maternal and fetal aspects of the placenta were graded, there was a correlation between clinical condition and scores for placental inflammation, with mean scores of 5.5 for 'low-suspicion BS,' 8.186 for 'equivocal BS' and 12.214 for 'highsuspicion BS' (see Fig.2). This suggests that the more severe the placental inflammation, the more likely it is to represent a clinically significant infection. However, the spread of scores in each group limits the utility of this scoring system in individual cases.

\section{Conclusion}

In a cohort of women managed for intrapartum sepsis during labour, $94.6 \%$ had normal PCT levels at the time of the septic screen and $58.5 \%$ had normal serial PCTs. The low number of women with a positive PCT was consistent with the low number with confirmed bacteraemia. This provides a basis that PCT may complement clinical judgement and interpretation of already utilised prognostic and diagnostic tests, in order to improve patient care in the management of intrapartum sepsis. Serial PCT measurements may be useful in predicting the prognosis, monitoring patients and tailoring therapy to individual needs of patients (antibiotic stewardship). Further research is required to determine the sensitivity and specificity of PCT as a biomarker for suspected bacterial sepsis in labour.

Further research is required to determine whether scoring of placental inflammation could be usefully interpreted in individual cases, prior to widespread adoption into routine clinical reporting.

\section{Acknowledgements}

Victoria Bills (Consultant Obstetrician at UHBW) who helped with the study design.

Melanie Griffin (Consultant Obstetrician at UHBW) who helped with the study design.

Andrew Day (Consultant in Clinical Biochemistry at UHBW) who helped with the PCT assay.

Philip Williams (Consultant Microbiologist at UHBW) who helped collect the blood culture data.

\section{Disclosures of Interests}

None declared. Completed disclosure of interests form available to view online as supporting information.

\section{Contribution to authorship}

SW helped design the study, supervised the data collection and drafted the article.

IH helped design the study and revised the article for intellectual content.

KS performed the statistical analysis of the data.

ARB reported the placental histopathology.

SC and AHW helped with data collection.

REL supervised the study, designed the study and revised the article for intellectual content.

\section{Details of ethics approval}

The hospital research ethics committee approved the study protocol (date of approval $=9^{\text {th }}$ June 2020). 


\section{Funding}

This pilot project was supported by grant from the local obstetric department. The funders played no role in data analysis, interpretation of findings or the decision to submit the manuscript for publication.

\section{References:}

1. Greer O, Shah NM, Johnson MR. Maternal sepsis update: current management and controversies. The Obstetrician and Gynaecologist. 2019;22(1):45-55

2. Tujula B, Kokki H, Rasanen J, Kokki M. Procalcitonin; a feasible biomarker for severe bacterial infections in Obstetrics and Gynecology? Acta Obstetricia et Gynecologica Scandinavica. 2018;97(5):505506

3. National institute for health and care excellence. Procalcitonin testing for diagnosing and monitoring sepsis (ADVIA Centaur BRAHMS PCT assay, BRAHMS PCT sensitive Kryptor assay, Elecsys BRAHMS PCT assay, LIASON BRAHMS PCT assay and VIDAS BRAHMS PCT assay). Diagnostic guidance 18. London: NICE; 2015 [http://www.nice.org.uk/guidance/dg18]

4. Agarwal R, Priyadarshini P, Mehndiratta M. Serum procalcitonin in pregnancy-associated sepsis: a case control study. South African Journal of Obstetrics and Gynaecology. 2019;25(1):15-19

5. Velasquez JESUS, Zuleta J, Portilla P, Caicedo K, Portilla L, Patino J. Usefulness of measuring procalcitonin (PTC) in pregnancy for the initial diagnosis of bacterial infection with systemic features. American Journal of Obstetrics and Gynecology. 2018;218(1):514-515

6. Elecsys BRAHMS Procalcitonin assay. Electrochemiluminescence immunoassay "ECLIA" for the in vitro quantitative determination of PCT (procalcitonin) in human serum and plasma. Found at: https://diagnostics.roche.com/global/en/products/params/elecsys-brahmsprocalcitonin-pct.html. AND: https://www.nortonsoundhealth.org/wp-content/uploads/ElecsysBRAHMS-PCT.pdf

7. Wood SL, Montoya A, Barreix M, Pi L, Calvert C, Rehman AM, et al. Incidence of maternal peripartum infection: A systematic review and meta-analysis. PLOS Medicine. 2019;16(12): e1002984

8. Tita A, Andrews W. Diagnosis and management of clinical chorioamnionitis. Clinics in Perinatology. 2010;37(2):339-354

9. Royal College of Obstetricians and Gynaecologists. Bacterial Sepsis in Pregnancy. Green-top Guideline No. 64a. London: RCOG; 2012

10. National institute for health and care excellence. Intrapartum care for women with existing medical conditions or obstetric complications and their babies. NICE guideline 121. 2019

11. Towers CV, Yates A, Zite N, Smith C, Chernicky L, Howard B. Incidence of fever in labor and risk of neonatal sepsis. The American Journal of Obstetrics and Gynaecology. 2017;216(6):596

12. Burgess APH, Katz JE, Moretti M, Lakhi N. Risk factors for intrapartum fever in term gestations and associated maternal and neonatal sequelae. Gynaecologic and Obstetric Investigation Journal. 2017;82(5):508-516

13. Coker MO, Hoen AG, Dade E, Lundgren A, Li Z, Wong AD, et al. Specific class of intrapartum antibiotics relates to maturation of the infant gut microbiota: a prospective cohort study. BJOG: an international journal of obstetrics and Gynaecology. 2020;127(2):217-227

14. Tapiainen T, Koivusaari P, Brinkac L, Lorenzi HA, Salo J, Renko M, et al. Impact of intrapartum and postnatal antibiotics on the gut microbiome and emergence of antimicrobial resistance in infants. Scientific Reports. 2019;9(10635) https://doi.org/10.1038/s41598-019-46964-5

15. Vijayan AL, Vanimaya, Ravindran S, Saikant R, Lakshmi S, Kartik R, et al. Procalcitonin: a promising diagnostic marker for sepsis and antibiotic therapy. Journal of Intensive care. 2017;5(51). https://doi.org/10.1186/s40560-017-0246-8

16. Schuetz P, Beishuizen A, Broyles M, Ferrer R, Gavazzi G, Gluck EH, et al. Procalcitonin (PCT)guided antibiotic stewardship: an international experts' consensus on optimized clinical use. Clinical Chemistry and Laboratory Medicine. 2019;57(9). doi.org/10.1515/cclm-2018-1181

17. Redline RW, Faye-Petersen O, Heller D, Qureshi F, Savell V, Vogler C. Amniotic infection syndrome: nosology and reproducibility of placental reaction patterns. Pediatric and Developmental Pathol- 
ogy.2003;6(5):435-448

18. Khong TY, Mooney EE, Ariel I, Balmus NCM, Boyd TK, Brundler MA, et al. Sampling and Definitions of Placental Lesions; Amsterdam Placental Workshop Group Consensus Statement. Archives of Pathology and laboratory medicine. 2016;140(7):698-713

19. Albright CM, Ali TN, Lopes V, Rouse DJ, Anderson BL. The Sepsis in Obstetrics Score: a model to identify risk of morbidity from sepsis in pregnancy. American Journal of obstetrics and gynaecology. 2014;211(39):1-8

20. Kuzniewicz MW, Walsh EM, Li S, Fischer A, Escobar GJ. Development and Implementation of an Early-onset sepsis calculator to guide Antibiotic Management in Late Preterm and Term Neonates. The Joint Commission Journal on Quality and Patient Safety. 2016;42(5):232-239

21. Neonatal Early-Onset Sepsis Calculator. Kaiser Permanente Research. Found at: https://neonatalsepsiscalculator.kaiserpermanente.org

22. Samantha C, Miller H, Leonard SA, Datoc IA, Girsen AI, Kappagoda S, et al. Lactate and procalcitonin levels in peripartum women with intraamniotic infection. American Journal of Obstetrics and Gynaecology. 2021;3(4):100367

23. Gregoriano C, Heilmann E, Molitor A, Schuetz P. Role of Procalcitonin use in the management of sepsis. Journal of Thoracic Disease. 2020; Feb (12):5-15

24. Rhee C. Using Procalcitonin to guide Antibiotic Therapy. Open Forum Infection diseases. 2016;4(1): https://doi.org/10.1093/ofid/ofw249

25. Hu R, Han C, Pei S, Mingzhu Y, Chen X. Procalcitonin levels in COVID-19 patients. International Journal of antimicrobial agents. 2020;56(2):10651

26. Peters C, Williams K, Un EA, Little L, Saad A, Lendrum K, et al. Use of procalcitonin for antibiotic stewardship in patients with COVID-19: A quality improvement project in a district general hospital. Clinical Medicine Journal. 2021: https://doi.org/10.7861/clinmed.2020-0614

27. Dockree S, Brook J, James T, Shine B, Vatish M. A pregnancy-specific reference interval for procalcitonin. Clinical Chimica Acta Journal. 2021; 513:13-16

28. Joyce CM, Deasy S, Abu H, Lim YY, O'Shea PM, O'Donaghue K. Reference values for C-reactive Protein and procalcitonin at term pregnancy and in the early postnatal period. Annals of Clinical Biochemistry. 2021;58(5):452-460

29. Lee J, Bang YH, Lee EH, Choi BM, Hong YS. The influencing factors on procalcitonin values in newborns with noninfectious conditions during the first week of life. 2017;60(1):10-16

30. Coburn B, Morris AM, Tomlinson G, Detsky AS. Does this adult patient with suspected bacteremia require blood cultures? The Journal of the American Medical Association. 2012;308(5):502-11

31. Schuetz P, Mueller A, Trampuz A. Serum procalcitonin for discrimination of blood contamination from bloodstream infection due to coagulase-negative staphylococci. Journal of Infection. 2007;35(5):352-5

32. Laukemann S, Kasper N, Kulkarni P, Steiner D, Rast AC, Kutz A, et al. Can we reduce negative blood cultures with clinical scores and blood markers? Results from an observational cohort study. Medicine. 2015;94(49):e2264

33. Jessop F, Sebire NJ. Histological chorioamnionitis: current concepts of diagnosis, classification and clinical significance. Fetal and Maternal Medicine Review. 2011;22(1):25-44

34. Smulian JC, Shen-Schwarz S, Vintzileos AM, Lake MF, Ananth CV. Clinical chorioamnionitis and histologic placental inflammation. Obstet Gynecol. 1999 Dec;94(6):1000-5

35. Goldstein JA, Gallagher K, Beck C, Kumar R, Gernand AD. Maternal-Fetal Inflammation in the Placenta and the Developmental Origins of Health and Disease. Front Immunol. 2020;13(11):531543

36. Roberts DJ, Celi AC, Riley LE, Onderdonk AB, Boyd TK, Johnson LC, et al. Acute Histologic Chorioamnioniits at Term: Nearly Always Noninfectious. Public Library of science. 2012;7(3):e31819

\section{Hosted file}

Figure 1.docx available at https://authorea.com/users/455982/articles/553186-an-evaluationinto-the-use-of-procalcitonin-levels-as-a-biomarker-of-bacterial-sepsis-to-aid-the-

management-of-intrapartum-pyrexia-and-chorioamnionitis-a-prospective-observational- 
cohort-study

\section{Hosted file}

Figure 2.docx available at https://authorea.com/users/455982/articles/553186-an-evaluationinto-the-use-of-procalcitonin-levels-as-a-biomarker-of-bacterial-sepsis-to-aid-themanagement-of-intrapartum-pyrexia-and-chorioamnionitis-a-prospective-observationalcohort-study 